\title{
James Webb Space Telescope Optical Telescope Element Mirror Coatings
}

\author{
Ritva. A. Keski-Kuha, Charles W. Bowers, Manuel A. Quijada \\ NASA/Goddard Space Flight Center, Greenbelt, Maryland 20771 \\ James B. Heaney \\ SGT Inc. Greenbelt, Maryland 20770 \\ Benjamin Gallagher \\ Ball Aerospace \& Technologies Corp., 1600 Commerce Street, Boulder, CO 80301 \\ Andrew McKay \\ Northrop Grumman Aerospace Systems, Redondo Beach, California \\ Ian Stevenson \\ Quantum Coating Inc., Moorestown, NJ 08057
}

\begin{abstract}
James Webb Space Telescope (JWST) Optical Telescope Element (OTE) mirror coating program has been completed. The science goals of the JWST mission require a uniform, low stress, durable optical coating with high reflectivity over the JWST spectral region. The coating has to be environmentally stable, radiation resistant and compatible with the cryogenic operating environment. The large size, $1.52 \mathrm{~m}$ point to point, light weight, beryllium primary mirror (PM) segments and flawless coating process during the flight mirror coating program that consisted coating of $21 \mathrm{flight}$ mirrors were among many technical challenges. This paper provides an overview of the JWST telescope mirror coating program. The paper summarizes the coating development program and performance of the flight mirrors.
\end{abstract}

Key words: James Webb Space Telescope, Optical Telescope Element, OTE, Mirrors, Coating, Reflectivity.

\section{INTRODUCTION}

James Webb Space Telescope ${ }^{1}$ Optical Telescope Element (OTE) $)^{2.3 .4}$ is a three mirror anastigmat consisting of a $6.5 \mathrm{~m}$ primary mirror $(\mathrm{PM})$, secondary mirror $(\mathrm{SM})$ and a tertiary mirror ${ }^{\mathrm{TM}}$. The primary mirror $(\mathrm{PM})$ is made out of 18 segments. The substrate material for the primary mirror segments as well as the SM, the TM and the fine steering mirror (FSM) is beryllium. A key factor in optimizing the throughput of FWST is the selection of the optical coating for the mirrors. The operating wavelength range is the key consideration in selecting the coating. To maximize throughput, the reflectivity of the coating should be as high as possible over the operational wavelength range. Additionally, the coating process and materials have to be compatible with the size of the optic and the substrate material. The coating needs to survive the environmental conditions the telescope is exposed to on the ground for several years before launch, as well as the 10 year lifetime on orbit.

Protected gold was selected as the coating for the JWST telescope mirrors. It provides high reflectivity, robust coating for applications above $600 \mathrm{~nm}$ through the IR covering the wavelength range of JWST of $800 \mathrm{~nm}$ to $29 \mu \mathrm{m}$. Protected silver coating was considered for the JWST telescope mirrors; however, protected silver is not as robust as protected gold. Protected silver is a commonly used coating in applications where high reflectivity is required down to $400 \mathrm{~nm}$. Since JWST's science does not require high throughput below $800 \mathrm{~nm}$, protected gold was selected over protected silver.

\section{COATING QUALIFICATION PROGRAM}

Quantum Coating Inc. (QCI) $)^{5}$ provided the coatings for the JWST telescope mirrors. Due to the large size of the primary mirror segments QCI designed and built a new state of the art coating facility for coating the JWST telescope optical 
components. This includes a new large coating chamber with access for mirror installation and removal from a cleanroom and another cleanroom for staging the optical components to be coated. After completion of the chamber build and commissioning QCI developed a protected gold coating process for the new chamber that produced high quality, high reflectivity coatings with performance similar to what they achieve in their smaller chambers. Once the new chamber was qualified to produce high quality coatings an extensive protective gold coating qualification program for JWST telescope beryllium mirrors started. Figure 1 shows the relative sizes of the PM segments, the SM and the TM. The table in figure 1 shows the sizes of the mirrors to be coated.

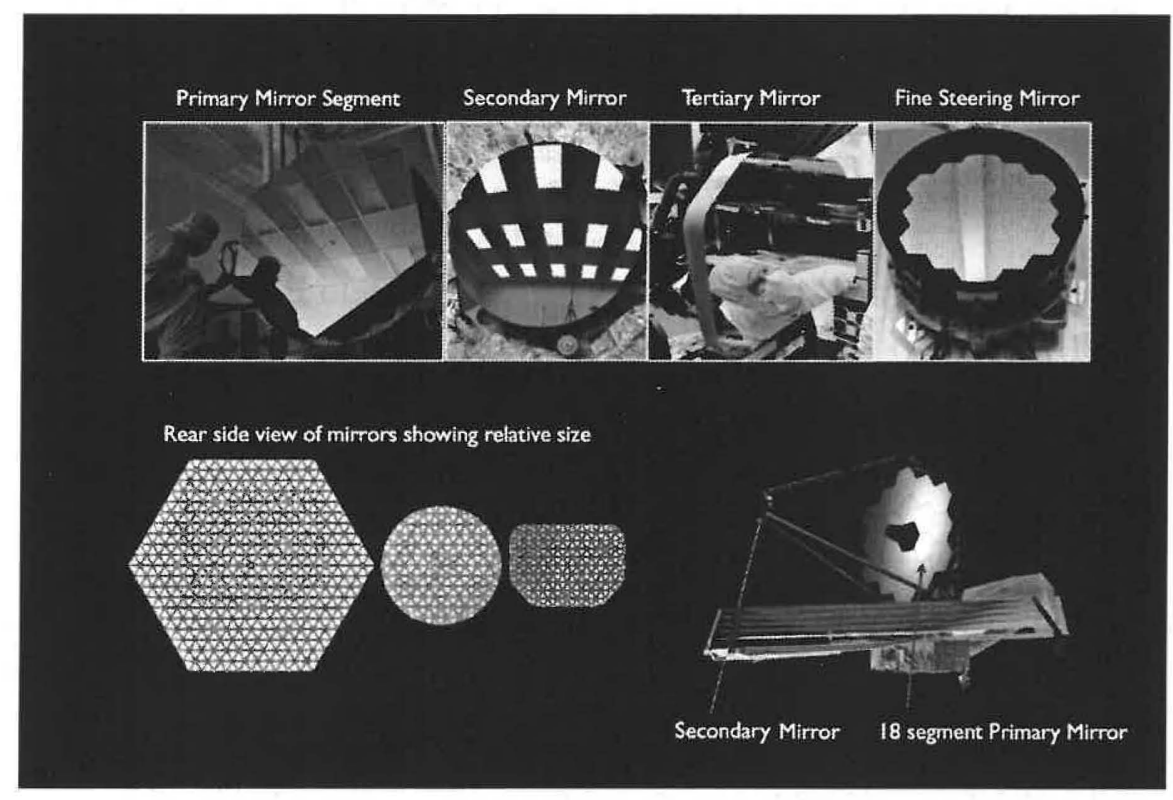

\begin{tabular}{|l|l|l|}
\hline Mirror & Size & Figure \\
\hline Primary Mirror & $6.5 \mathrm{~m}$ aperture f/1.2 & Elliptical \\
\hline PM Segments & $1.52 \mathrm{~m}$ point to point & \\
\hline Secondary Mirror & $0.738 \mathrm{~m}$ diameter & Hyperbolic \\
\hline Tertiary Mirror & $0.513 \mathrm{~m} \mathrm{x} 0.709 \mathrm{~m}$ & Elliptical \\
\hline Fine Steering Mirror & $0.17 \mathrm{~m}$ diameter & Flat \\
\hline
\end{tabular}

Figure 1. JWST. telescope mirrors.

The qualification program verified that QCI coating process, procedures and the new chamber reliably produced coatings that met all JWST mirror coating requirements. This included reflectance, thickness uniformity across each type of mirror, coating stress, durability, micro roughness, processing high temperature limit, coating maintainability, run to run thickness variation in case of the primary mirror segments, survival after exposure to extreme temperatures and radiation resistance. The coating process had to robust to reliably coat all 21 flight mirrors without a failure because the coating could not be stripped off. It could only be removed by grinding it off and re-polishing the mirror.

JWST Primary Mirror Surrogate Segment with openings for both beryllium and glass witness samples was used for the qualification runs for the primary mirror segments. Be coupons, 1 " in diameter were used to qualify reflectance, durability, maintainability and micro roughness. Uniformity and run to run thickness variation was verified with lines of glass slides across the surrogate that allowed for denser uniformity data. Two inch diameter glass wafer stress coupons were also included for qualification runs to qualify the coating stress. The first few qualification runs included an 8 " diameter glass flat. It was used for evaluation of the scratch/dig and pinhole requirements. The last qualification run included an 8" diameter Be coupon to qualify durability, scratch-dig, coating stress, and maintainability. 


\subsection{Results}

\section{Reflectance}

Table 1 shows the reflectivity requirements for the mirror coating. This ensures that the science goals can be achieved even with some loss in reflectance due to contamination over the lifetime of the telescope.

\begin{tabular}{|l|l|}
\hline Wavelength $(\mu \mathrm{m})$ & Reflectance Requirements (\%) \\
\hline 0.8 & $\geq 94.1$ \\
\hline 1.0 & $\geq 96.3$ \\
\hline 1.5 & $\geq 97.4$ \\
\hline $2.0-20.0$ & $\geq 97.9$ \\
\hline $20.1-27.0$ & $\geq 97.9$ \\
\hline $27.1-29.0$ (Goal) & $\geq 97.9$ \\
\hline
\end{tabular}

Table 1. Reflectance requirements.

To qualify coating reflectivity and uniformity across the clear aperture of a mirror, reflectance was measured on optical witness samples coated during coating qualification runs. Witness samples were distributed across the clear aperture of each mirror type using surrogates and outside the clear aperture in locations where the witness samples were located during the final mirror coating run. These outside of clear aperture witness samples verified that they were representative of the coating performance on the mirrors. The reflectance from $800 \mathrm{~nm}$ to $2500 \mathrm{~nm}$ was measured at QCI using visibie/near infrared spectrometer at $6^{\circ}$ angle of incidence with spectral resolution of $1 \mathrm{~nm}$ and from $2500 \mathrm{~nm}$ to $20 \mu \mathrm{m}$ at $10^{\circ}$ using an FTIR spectrometer with spectral resolution of $5 \mathrm{~nm}$.

The operating temperature range for the telescope mirrors is $20 \mathrm{~K}$ to $55 \mathrm{~K}$. To verify that the reflectivity meets requirements at cryogenic operating temperatures, reflectance of two protected gold coated Be samples, prepared by QCI during the coating qualification program were measured over the wavelength range from $0.3 \mu \mathrm{m}$ to $40 \mu \mathrm{m}$, and at selected temperatures over the $300 \mathrm{~K}$ to $35 \mathrm{~K}$ range. Visual inspection was also performed at the end of each cryo cycle. These measurements were made at Goddard Space Flight Center (GSFC).

For the $0.3 \mu \mathrm{m}$ to $3.0 \mu \mathrm{m}$ wavelength range a spectrophotometer with LN2 cryostat cryo-reflectometer attachment was used. It can maintain sample temperature to better than $\pm 1 \mathrm{~K}$. The angle of incidence was $8^{\circ}$, spectral resolution $1 \mathrm{~nm}$ and temperature range $300 \mathrm{~K}-77 \mathrm{~K}$.

For the $2.0 \mu \mathrm{m}$ to $40.0 \mu \mathrm{m}$ wavelength range Bruker Fourier Transform Spectrometer was used. The sample temperature can be controlled to better than $\pm 0.5 \mathrm{~K}$ over the $300 \mathrm{~K}$ to $35 \mathrm{~K}$ temperature range. The wavelength range of the instrument is $0.5 \mu \mathrm{m}$ to $40 \mu \mathrm{m}$, spectral resolution $2 \mathrm{~cm}^{-1}$ and the angle of incidence $8^{\circ}$. Reflectance over the $0.3 \mu \mathrm{m}$ to $40 \mu \mathrm{m}$ is measured relative to an evaporated gold mirror standard.

Figure shows measured reflectance up to $20 \mu \mathrm{m}$. The measured data show no change in reflectance over this wavelength and temperature regime, except for a very slight indication $(\sim 0.01)$ of a reflectance increase in the $0.60 \mu \mathrm{m}$ to $1.0 \mu \mathrm{m}$ wavelength region where the gold spectrum transitions through the high-low plasma frequency region. The results between $20 \mu \mathrm{m}$ and $40 \mu \mathrm{m}$ were the same. A non-contact visual inspection detected no change in coating appearance after each cryo-cycle. 


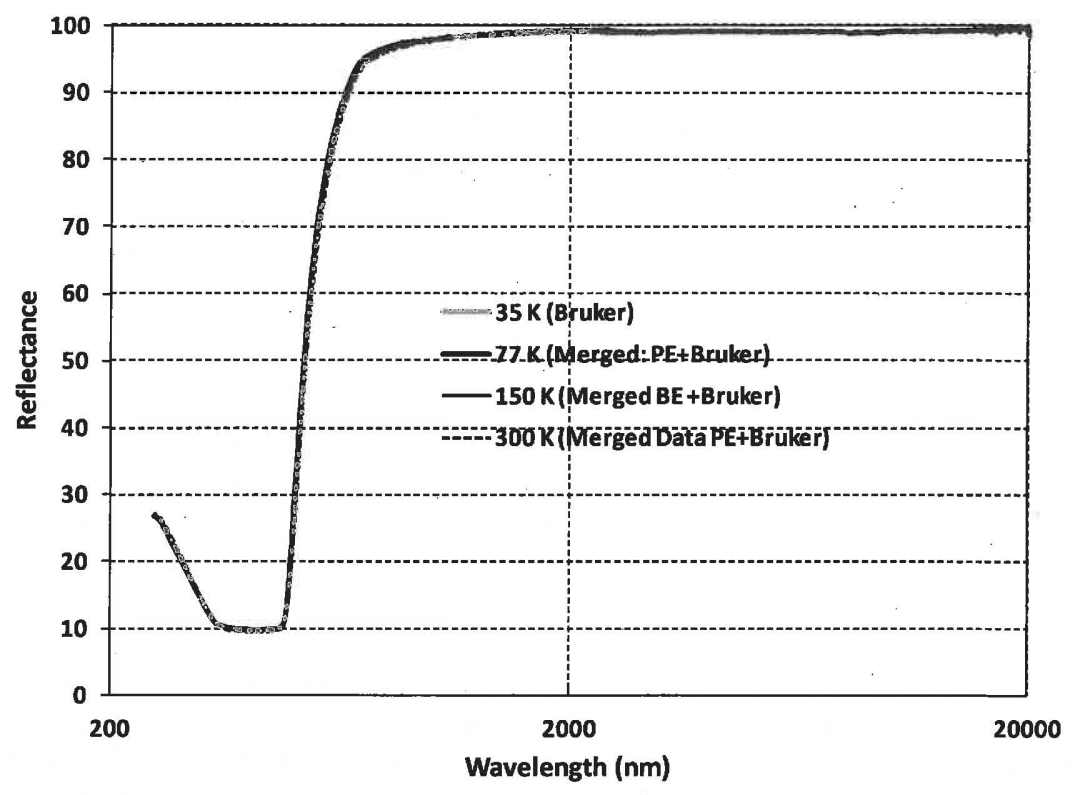

Figure 2. The reflectance of the QCI protected gold sample over the wavelength range from $250 \mathrm{~nm}$ to $20,000 \mathrm{~nm}(0.25 \mu \mathrm{m}$ to $20 \mu \mathrm{m})$ and at temperatures from $300 \mathrm{~K}$ to $35 \mathrm{~K}$.

\section{Radiation Exposure}

The objective of the irradiation exposure test of the JWST OTE mirror coatings is to measure the change in coating reflectance as a function of exposure to a simulated JWST solar wind orbital radiation environment. Four test samples, coated by QCI during coating qualification were exposed in the Goddard Space Flight Center's Solar Wind Facility (SOWF) ${ }^{6}$ to an approximate L2 orbital electron and proton environment. Reflectance measurements were made in-situ before, during, and after the radiation exposure.

QCI applied their protected gold coating formulation directly onto the polished bases of Al substrates that are shaped like "chess pawns" to accommodate the mechanical grabber fixture in the SOWF that transfers samples from the irradiation chamber into the integrating sphere reflectometer while remaining in the same vacuum environment of $\sim 10^{-7}$ Torr. Although the actual JWST OTE flight mirrors will use Be substrates, the choice of substrate material does not affect the irradiation test results, because the low energy $(<10 \mathrm{keV})$ electrons and protons used in this test do not penetrate through to the samples' Al substrates. The reflectance measurements, that produce the test results, sample through the dielectric overcoat but do not penetrate through the gold layer and never sample the substrate material. Consequently, in this test configuration, the substrate choice is irrelevant.

According to plan, the radiation exposure was conducted in two stages. During stage 1 the 4 samples were exposed to 3 keV protons, only; no electrons. The proton exposure rates for each sample, as monitored by the Faraday cups located adjacent to the samples in the mounting plate are presented in the table 2. The duration of each exposure, in seconds, is also shown in the table, corresponding to the intervals after which sample reflectance was measured. The total fluence is displayed with the reflectance measurements. In stage 2 the 4 samples were exposed to combined beams of $3 \mathrm{keV}$ protons and $10 \mathrm{keV}$ electrons. The proton and electron exposure rates are also presented in table 2. 


\begin{tabular}{|c|c|c|c|c|c|}
\hline Proton Beam Energy: & 3.0KeV & & & & \\
\hline \multirow[t]{4}{*}{ Electron Beam Energy: } & $10 \mathrm{KeV}$ & & & & \\
\hline & \multicolumn{5}{|c|}{ Exposure Rates for JWST OTE Gold-Coated Test Samples } \\
\hline & $\begin{array}{c}\text { First Exposure } \\
\text { time length } \\
\text { (sec) }\end{array}$ & $\begin{array}{c}\text { 2nd Exposure } \\
\text { time length (sec) }\end{array}$ & $\begin{array}{c}\text { 3rd Exposure time } \\
\text { length (sec) }\end{array}$ & \multicolumn{2}{|c|}{$\begin{array}{l}\text { 4th Exposure time } \\
\text { length (sec) }\end{array}$} \\
\hline & $2.38 E+05$ & $2.56 E+05$ & 2.55E+05 & \multicolumn{2}{|c|}{$2.62 E+05$} \\
\hline sample & $\mathrm{p} / \mathrm{cm}^{\wedge} 2 \mathrm{~s}$ & $\mathrm{p} / \mathrm{cm}^{\wedge} 2 \mathrm{~s}$ & $\mathrm{p} / \mathrm{cm}^{\wedge} 2 \mathrm{~s}$ & $\mathrm{p} / \mathrm{cm}^{\wedge} \mathbf{2 s}$ & $\mathrm{e} / \mathrm{cm}^{\wedge} 2 \mathrm{~s}$ \\
\hline G3 & $2.97 \mathrm{E}+09$ & $3.60 \mathrm{E}+09$ & $3.33 E+09$ & $2.21 \mathrm{E}+09$ & $2.30 E+09$ \\
\hline G4 & $2.84 \mathrm{E}+09$ & $3.50 \mathrm{E}+09$ & 3.17E+09 & $2.29 \mathrm{E}+09$ & $3.07 \mathrm{E}+09$ \\
\hline G6 & $2.98 \mathrm{E}+09$ & $3.55 \mathrm{E}+09$ & $3.29 \mathrm{E}+09$ & $2.29 E+09$ & $2.55 E+09$ \\
\hline G7 & $3.11 E+09$ & $3.67 E+09$ & $3.45 E+09$ & $2.18 \mathrm{E}+09$ & $1.81 E+09$ \\
\hline
\end{tabular}

Table 2. Proton and electron exposure rates for JWST OTE protected gold coated samples.

In addition to the Faraday cups, a $2 \mathrm{~mm}$ thick LiF crystal with an evaporated layer of $100 \mathrm{~nm}$-thick $\mathrm{Al}$ deposited onto its back surface, and a front surface $\mathrm{Ag}$ mirror with a dielectric overcoat were included in the radiation beam for environmental monitoring.

Figure 3 contain the SOWF measured reflectance of test sample G4. There was no measurable change in reflectance at the end of stages 1 and 2, therefore results of both stages are contained in the plot. The results for the other three samples were similar.

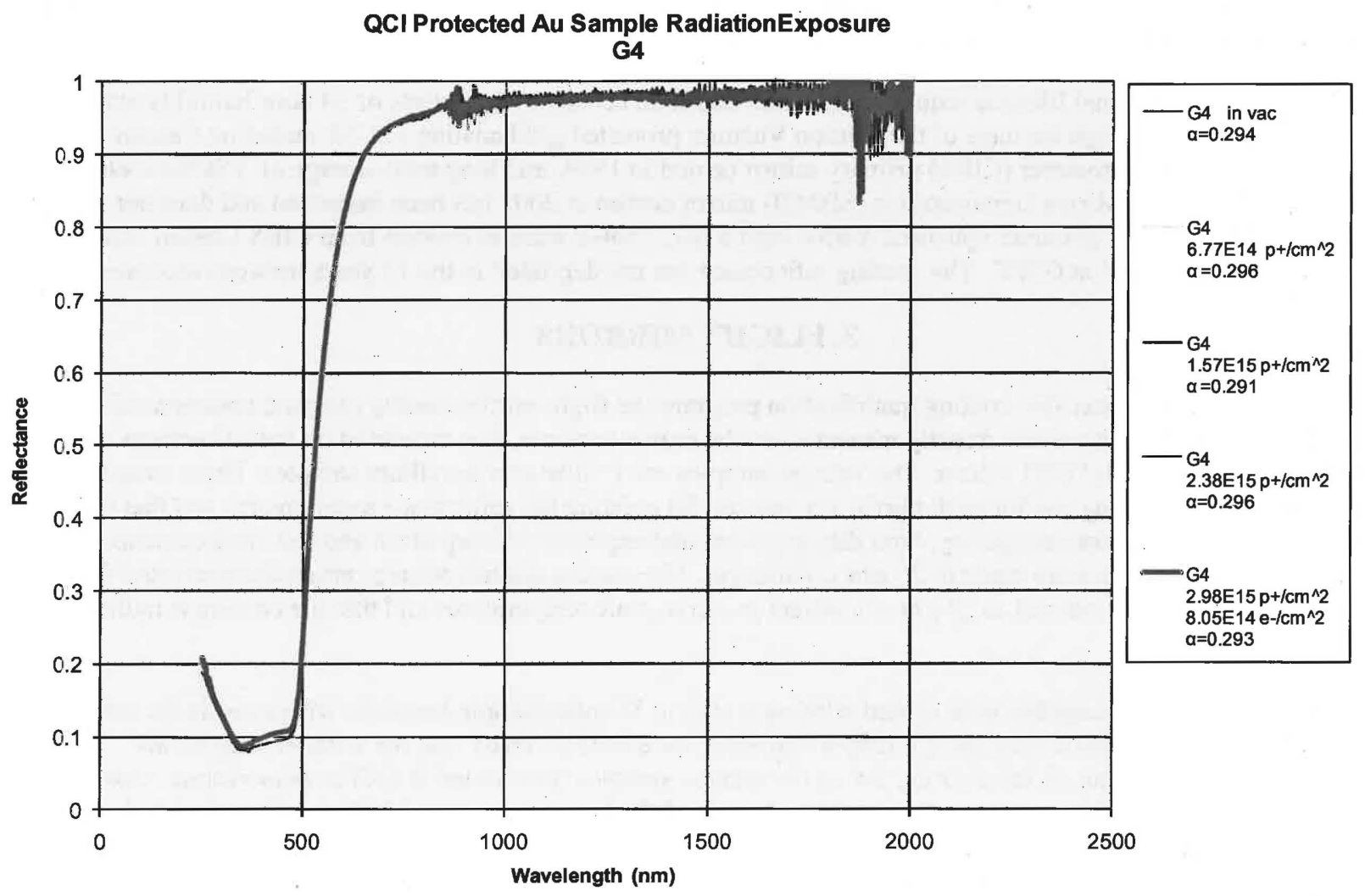

Figure 3. Reflectance of QCI sample G4 after exposure to $2.98 \mathrm{E} 15$ protons $/ \mathrm{cm}^{2}$ at $3 \mathrm{keV}$ plus $8.05 \mathrm{E} 14$ electrons $/ \mathrm{cm}^{2}$ at $10 \mathrm{keV}$ over the $250 \mathrm{~nm}$ to $2000 \mathrm{~nm}$ wavelength range. 
The JWST OTE samples that were coated with QCI's protected Au coating exhibited no change in reflectance within the $\sim \pm 0.003$ reflectance measurement precision after exposure to a dosage of $\sim 3.0 \times 10^{15}$ protons $/ \mathrm{cm}^{2}$ plus up to $8 \times 10^{15}$ electrons $/ \mathrm{cm}^{2}$. The environmental monitoring samples also exhibited no loss in reflectance. These results confirm that the QCI protected gold coating formulation is radiation stable up to the dosage used in this test.

\section{Other Requirements}

In addition to reflectance the extensive coating qualification program included qualification to non reflectance requirements, uniformity, surface roughness, stress and durability being the key features. The thickness uniformity across each mirror type was qualified using glass witness samples distributed across the clear aperture of each mirror type surrogate and in locations where the witness samples were located during a flight mirror coating run. Each sample was masked to create a step for thickness measurement. The coating met the $10 \mathrm{~nm}$ peak to valley uniformity requirement. The requirement for micro roughness was that it not degrade the surface roughness of the mirror. Micro roughness was qualified by measuring beryllium witness sample micro roughness before and after coating. The change for all samples measured was less than the noise in the measurement.

Coating stress was qualified using 2" diameter glass samples. There sag in the samples was measured before and after coating. The stress was calculated from the change in the sag. Three samples were included in each qualification run. The coating met the $10 \mathrm{ksi}$ stress requirements at ambient. The stress at cryogenic temperature was qualified using an $8^{\prime}$ diameter thin beryllium sample. The bending in the samples was measured at ambient and cryogenic temperature before and after coating. The results were in-line with modeling results.

Coating durability was qualified per MIL 13830B for humidity exposure, MIL 13830B for hardness and MIL 13830B for adhesion. QCI was also required to develop post coating cleaning procedure in case cleaning was ever needed before launch. The maintainability of the coating was qualified using 8 " diameter glass samples, silicon wafer samples and finally Be witness samples. Be samples were cleaned and then cryo cycled and tested for durability. Reflectance was measured before cleaning and after completion of all testing.

The over 10 year operational lifetime requirement is met based on accelerated life tests of 24 hour humidity exposure and cryo cycling, space flight heritage of the Denton Vacuum protected gold coating FSG98 including Cassini Composite Infrared Spectrometer (CIRS) primary mirror coated in 1996, and long term storage of FSG98 coated optics. The Subscale Beryllium Mirror Demonstrator (SBMD) mirror coated in 2001 has been inspected and does not show any degradation. This is $0.5 \mathrm{~m}$ diameter spherical mirror with a flat. Coated witness coupon from CIRS Cassini was inspected and re-measured at GSFC. The coating reflectance has not degraded in the 12 years between measurements.

\section{FLIGHT MIRRORS}

After completion of the extensive coating qualification program the flight mirror coating program commenced. For the flight mirrors the reflectance is not directly measured on the mirror surfaces. It is measured on optical witness samples (OWS) coated during each JWST mirror. The witness samples are $1^{\text {" diameter beryllium samples. These samples }}$ demonstrate that the coating run for each mirror was successful meeting the reflectance requirements and that the coating can stand cryogenic temperature cycling, humidity exposure and exposure to integration and test environments. Reflectance measurements were made to $20 \mu \mathrm{m}$ wavelength. The coating qualification program demonstrated that reflectance measurements are met to $29 \mu \mathrm{m}$ at ambient and cryogenic temperatures and that the coating is radiation stable.

Eleven beryllium witness samples were coated with each mirror. Witness sample locations were outside the mirror clear aperture. Coating qualification runs using a mirror surrogate were used to verify that the witness samples are representative of the coating on the mirrors. Six of the witness samples were tested at QCI to demonstrate coating performance. They were first cryogenically cycled to down to $15 \mathrm{~K}$ four times and to $328 \mathrm{~K}$ one time. To demonstrate survivability in humid environment they were exposed to a 50C temperature at $98 \%$ humidity for 24 hours. Reflectance was measured after completion of these tests. 
The other five samples were installed in the shipping container with the mirror. Upon arrival at Ball Aerospace two of these witness samples are stored in a dry box. The other three remained with the mirror to witness integration and test operations including cryogenic testing at Marshall Space Flight Center (MSFC) X-Ray and Cryogenic Facility (XRCF). After completion of the integration and test reflectance of one of these samples was measured to $20 \mu \mathrm{m}$. The final reflectance vs. wavelength curve for each mirror is the average of the six samples measured after cryo cycling and humidity testing and one of the samples that witnessed integration and test operations.

The first flight mirror to be coated was the Tertiary Mirror (TM). The coated aperture requirement was to within $7 \mathrm{~mm}$ of chamfer. This was achieved with masking which was qualified with TM surrogate mirror. During the TM coating run chamber circuit breakers that protected gold resistance source tripped causing multiple interruptions in TM gold layer deposition. Recovery plan during the coating run allowed the gold layer to reach critical thickness but the thickness was less than nominal. Reflectance was less than $1 \%$ below requirements at wavelengths below $2.5 \mu \mathrm{m}$. Coating stress was also slightly over the $10 \mathrm{ksi}$ limit. The coating passed all other requirements. After extensive witness sample testing to rule out lifetime and contamination issues and verifying that the coating is the same as qualification coatings with the exception of the thickness of the gold layer the mirror was accepted as is. Additionally TM reflection loss is made up with FSM coating and the throughput of the Aft Optics Assembly (AOS) consisting of the TM and FSM. The AOS will meet its throughput requirement.

Corrective actions were implemented before coating the Engineering Development Unit (EDU) primary mirror segment which is also the flight spare for primary mirror segment assembly (PMSA) type A. The mirror was tested at cryogenic temperature at XRCF after completion of final figuring at Tinsley and after coating to verify that the coating did not degrade the surface figure at cryogenic temperature. Figure 4 shows the TM and the EDU after completion of the coating.
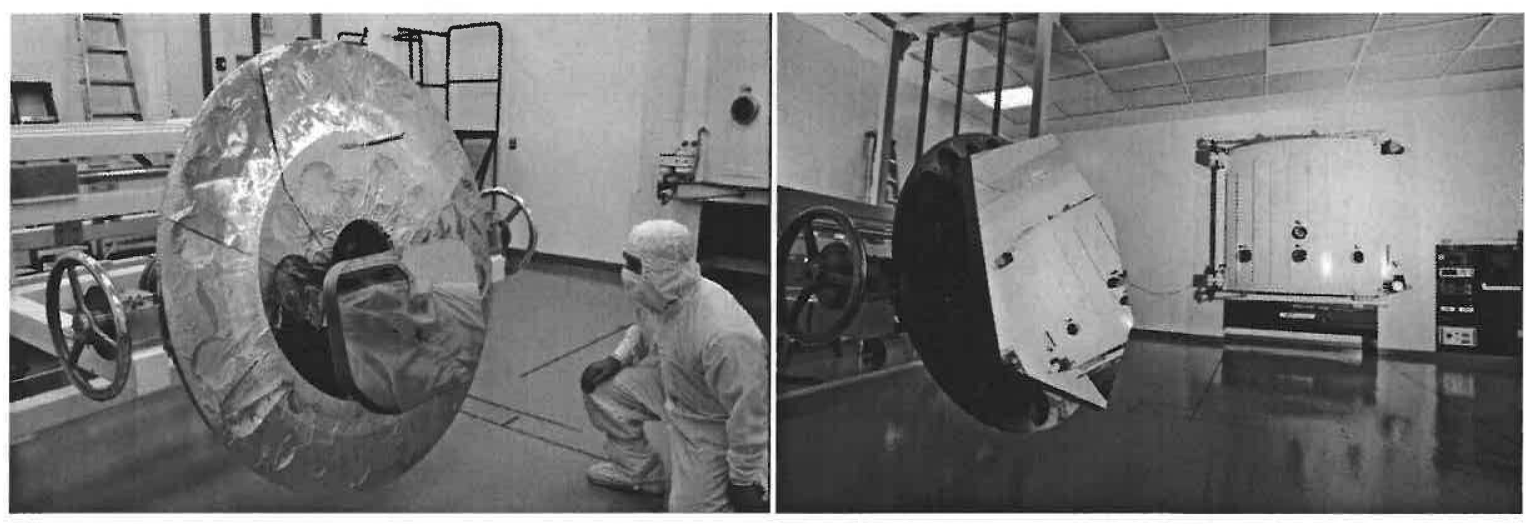

Figure 4. Tertiary mirror and the Engineering Design Unit (EDU) primary mirror segment, coated with gold by Quantum Coating Incorporated.

After completion of final figuring at Tinsley the 18 flight PMSAs, six segments each of type A, B and C, the secondary mirror and the fine steering mirror were sent to QCI for coating. The coating chamber was reconfigured for each mirror type and the layout qualified before coating a flight mirror. QCI processed the mirror assemblies in configuration 2 to protect the flight actuators from coating processes. In configuration 2 the hexapod assembly and the radius of curvature assembly were not attached. The coated aperture requirement for the PMSAs was to within $1 \mathrm{~mm}$ of the chamfer. This was achieved with masking that was qualified with mirror surrogates during the qualification program.

After the TM the flight mirror coating program was flawless. All flight mirrors were coated over approximately one year time period with excellent results. Figure 5 shows PMSA B6 in the coating fixture after completion of the coating and in the shipping container with the lid being installed. Figure 6 shows photos of PMSA C3 in the coating fixture after the coating with the mask in place, with mask removed, in the handling fixture being installed in the shipping container and in the shipping container with the lid being installed. The mirrors were shipped to Ball Aerospace for the actuator assembly installation and from there to XRCF at MSFC for the final figure verification at cryogenic temperature. The 
process was verified on the EDU. Figure 7 shows the coated secondary mirror. All SM, TM and FSM cryogenic temperature verification was done at Ball Aerospace.
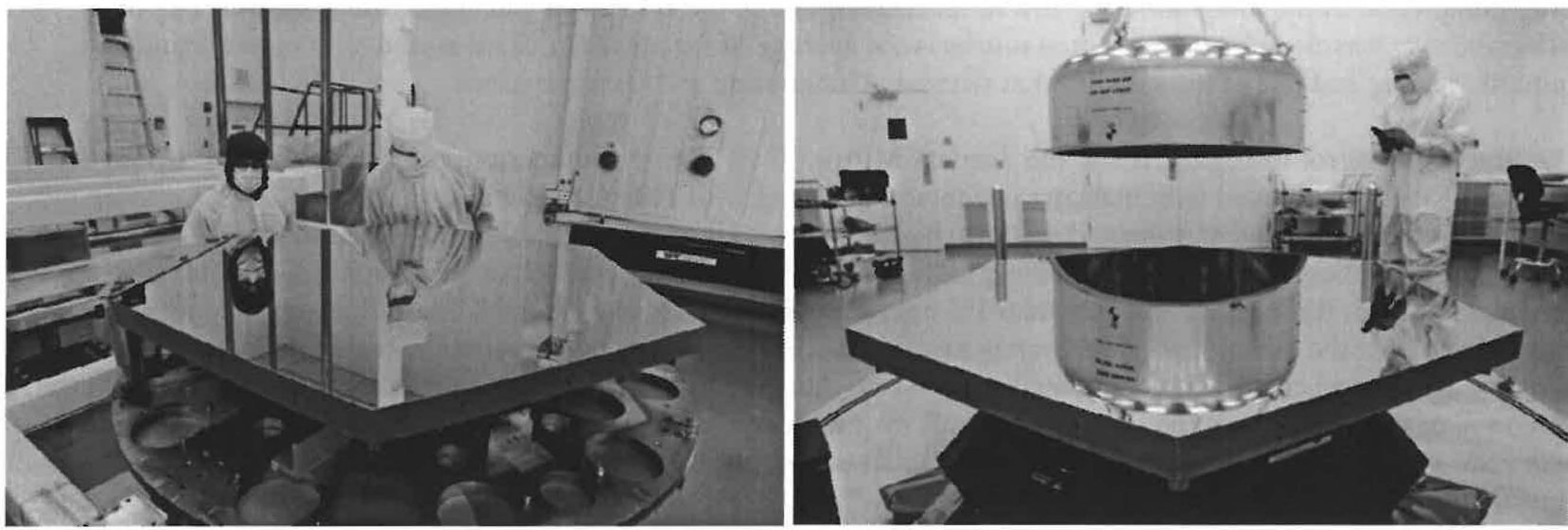

Figure 5. PMSA B6 in the coating fixture and being readied for shipment to BATC.
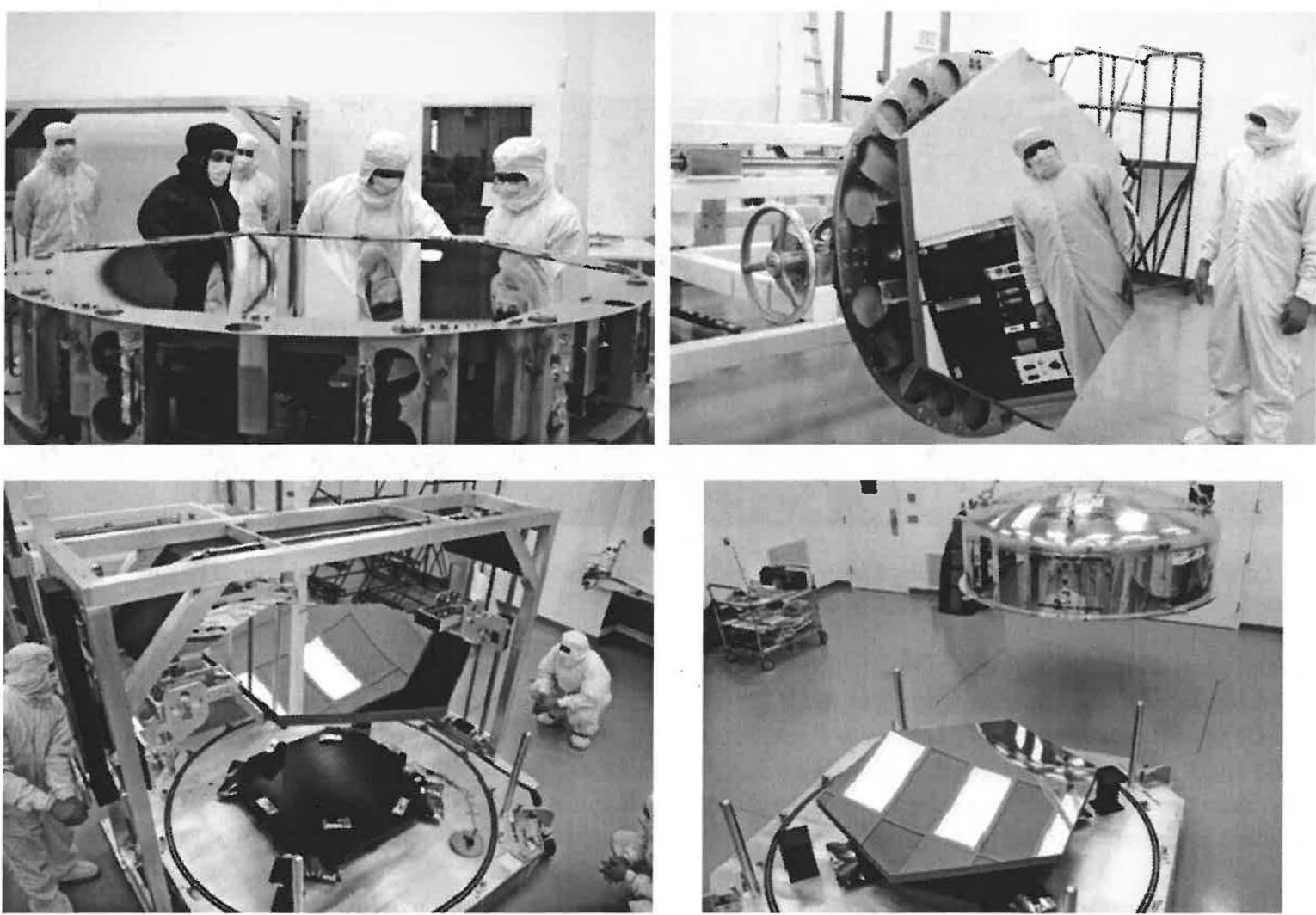

Figure 6. PMSA C3 in the coating fixture with the mask on, the mask removed (top pictures) and being readied for shipment to BATC. 


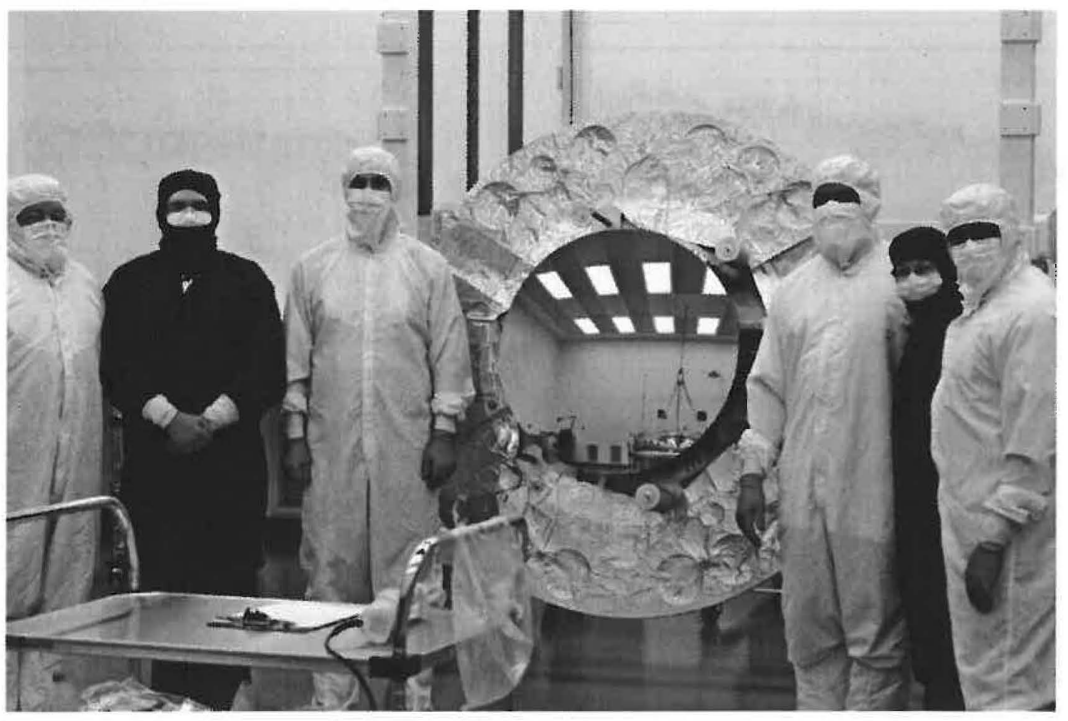

Figure 7. JWST telescope secondary mirror.

JWST mirror coating program achieved excellent results. Table 3 shows reflectance results for PMSA A1. Reflectance exceeds requirement at all wavelengths. Results for the other PMSAs, SM and FSM are similar.

\begin{tabular}{|l|l|l|}
\hline Wavelength $(\mu \mathrm{m})$ & Reflectance Requirement(\%) & Measured Reflectance (\%) \\
\hline 0.8 & $\geq 94.1$ & 96.0 \\
\hline 1.0 & $\geq 96.3$ & 97.3 \\
\hline 1.5 & $\geq 97.4$ & 98.0 \\
\hline $2.0-20.0$ & $\geq 97.9$ & $98.5-98.8$ \\
\hline $20.1-27.0$ & $\geq 97.9$ & $99.08-99.42$ \\
\hline $27.1-29.0$ (Goal) & $\geq 97.9$ & $99.16-99.35$ \\
\hline
\end{tabular}

Table 3. Measured reflectance for PMSA A1.

The reflectance of six acceptance witness samples coated with PMSA A1 is shown in Figure 8. These samples were measured after cryo cycling and humidity exposure testing. The $7^{\text {th }}$ sample witnessed PMSA A1 integration and test operations. Reflectance of this sample is similar to the other six samples. The results show that the integration and test operations did not degrade the coating performance. The final A1 reflectance is the average of these seven samples. Results for the other mirrors were similar. 

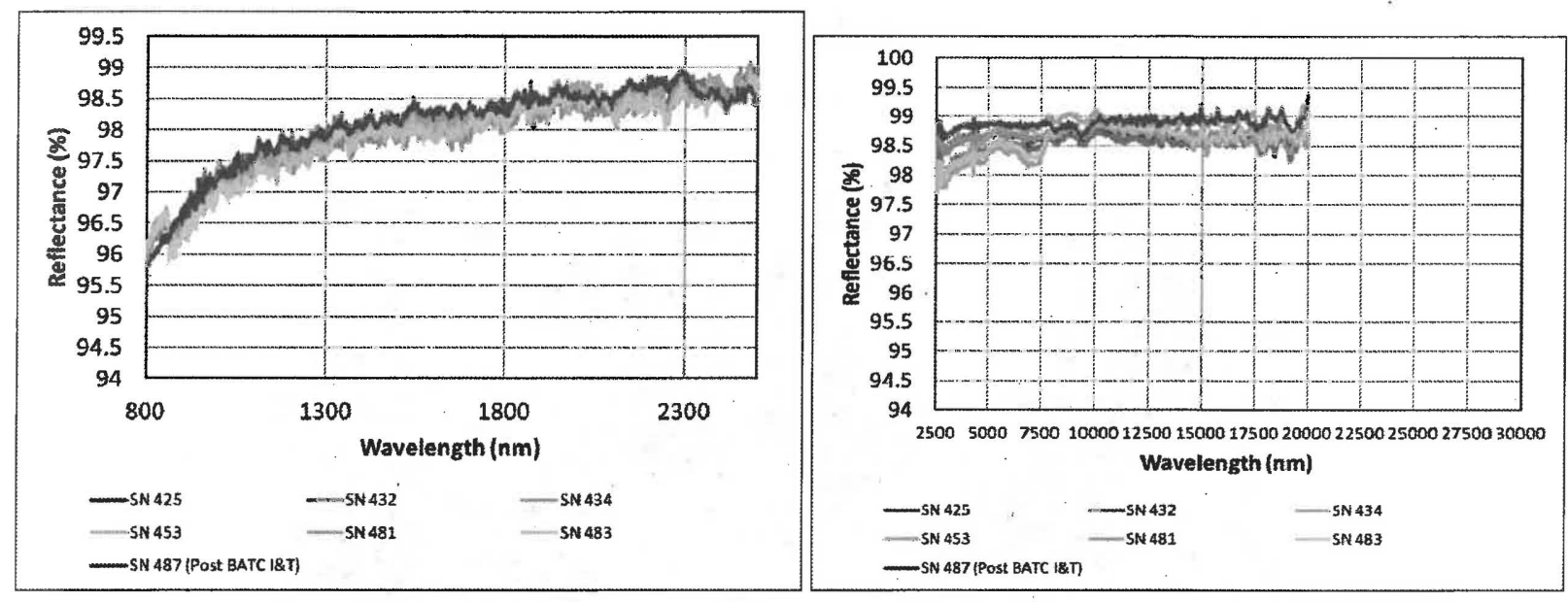

Figure 8. PMSA A1 Run Reflectance - Post BATC I\&T Sample compared to 6 QCI Acceptance Samples.

Figure 9. shows the average reflectivity of the 18 PMSA segments, SM, TM and FSM as well as the combined reflectivity of the telescope ${ }^{7}$. The reflectance requirement for individual mirrors as well as the combined telescope is shown for comparison. The results show that the telescope meets its reflectance requirements with margin providing excellent performance.

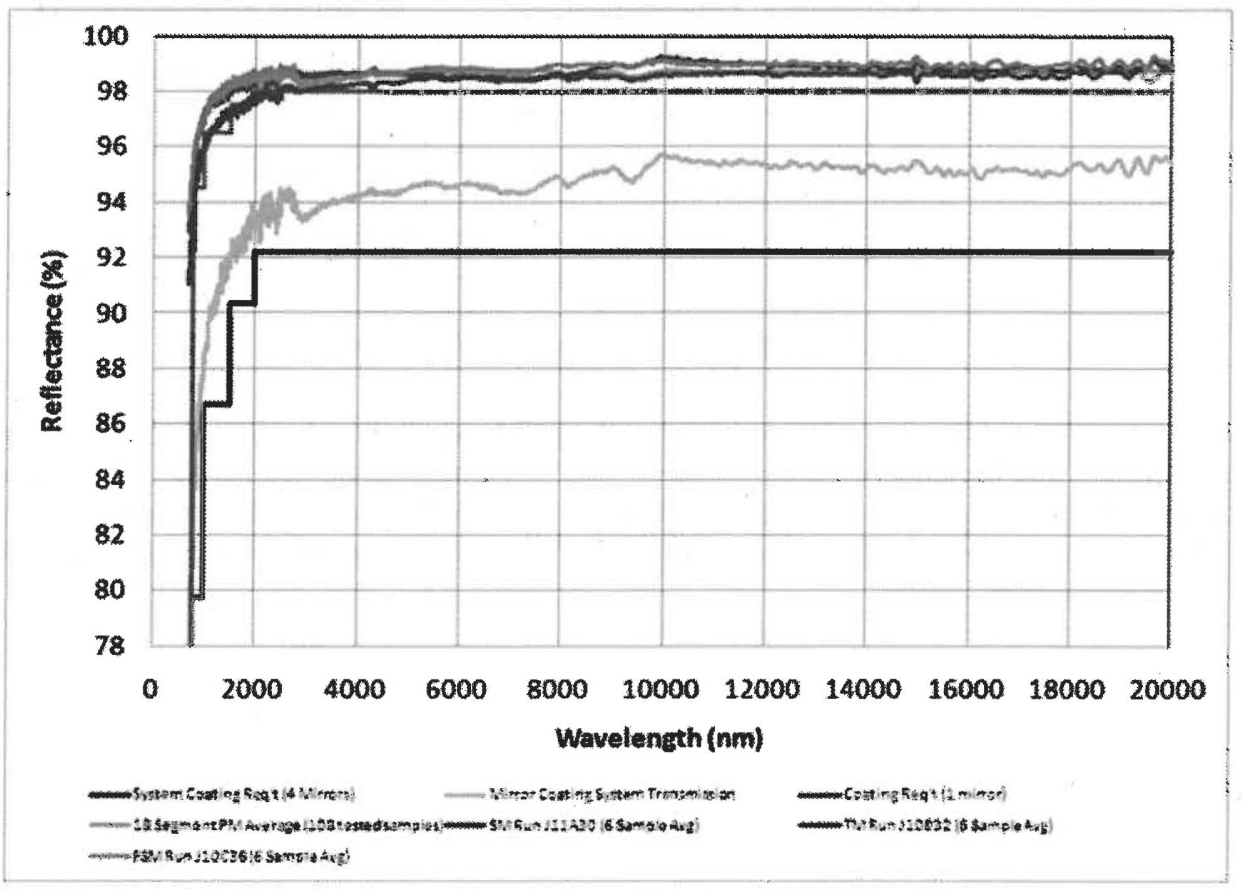

Figure 9. Spectral reflectivity of the JWST mirrors and the combined transmission through the telescope.

All 21 protected gold coated flight mirrors are shown in figure 10. All flight mirrors after the TM were coated without a single failure. This was an outstanding accomplishment. The coating cannot be stripped off. Therefore a failure during 
the coating process would have required grinding off the coating and re-polishing the mirror surface causing a major schedule delay.

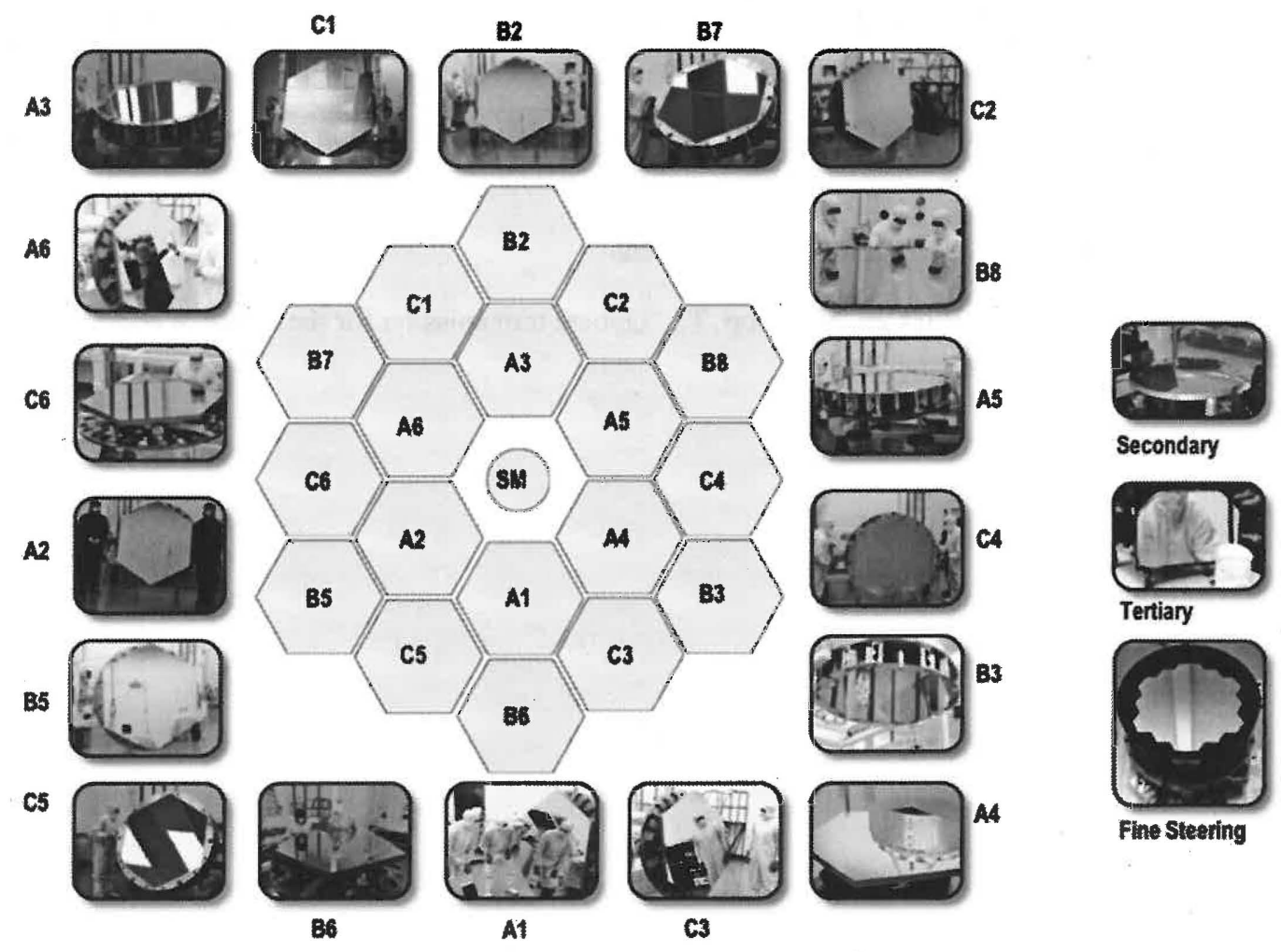

Figure 10. JWST Telescope Gold Coated Flight Mirrors.

\section{SUMMARY}

Completion of the JWST telescope mirror coating program was a major milestone in its development. The coating program was completed on schedule with excellent results. The large size of the JWST telescope primary mirror segments presented a major challenge. QCI constructed a new coating facility to produce a high reflectivity, uniform, low stress, environmentally stable coating on the $1.52 \mathrm{~m}$ primary mirror segments. An extensive coating development program over a two year period before coating the first flight mirror ensured that the coating met all JWST requirements. All the flight mirror coating runs had to be executed without a single failure. All the technical challenges were overcome. The coating easily met their specifications ensuring the scientific discovery potential of James Webb Space Telescope.

\section{REFERENCES}

1. Sabelhaus, P., Decker, J., “An Overview of the James Webb Space Telescope (JWST) Project”, Proceedings of SPIE, Vol. 5487, p 550-563 (2004).

2. Atkinson, C., Texter, S., Hellekson, R., Patton, K., Keski-Kuha, R., Feinberg, L., "Status of the JWST Optical Telescope Element", Proceedings of SPIE, Vol. 6265, 62650T (2006). 
3. Feinberg, L.D, Clampin, M., Keski-Kuha, R., Atkinson, C., Texter, S. "James Webb Space Telescope Optical Element Development History and Results”, Proc. SPIE 8442, (2012).

4. Atkinson, C, Texter, S., Keski-Kuha R., Feinberg L., "Status of the JWST Optical Telescope Element", Proc SPIE $8442,(2012)$.

5. Heaney, J.B, Kauder, , L.R, Bradley, S.F., and Neuberger, D., "Mirror degradation in orbit due to space radiation exposure", Proc. SPIE Vol. 4135, San Diego, (2000).

6. Quantum Coating Incorporated, www.quantumcoating.com.

7. Lightsey, P.A., Gallagher, B. B., Nickles, N., Copp, T., "Optical transmission for the James Webb Space Telescope", Proc. SPIE 8442, (2012). 